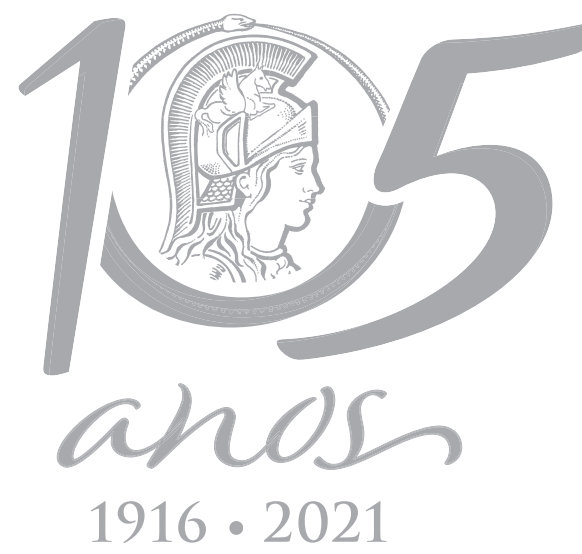

\title{
ECOSYSTEMS
}

\section{Species richness and distribution patterns of the snake fauna of Rio Grande do Norte state, northeastern Brazil}

\author{
RICARDO MARQUES, THAíS BARRETO GUEDES, FLÁVIA MOL LANNA, DANIEL \\ CUNHA PASSOS, WILLIANILSON PESSOA DA SILVA \& ADRIAN ANTONIO GARDA
}

\begin{abstract}
The Neotropics are one of the richest regions in biodiversity globally. Still, much remains unknown about the mechanisms and processes responsible for the accumulation of species in this region. Among the many limitations on our current knowledge on the region's biodiversity, understanding of community composition and species distributions is limited and greatly biased in many Brazilian regions. We present information on species composition, habitat use, geographic distribution, taxonomic accounts, and conservation of snakes from Rio Grande do Norte state, Northeastern Brazil. We compiled, from primary and literature data, 851 snake records from seven families and 47 species, with five new records for the state. Species are mainly terrestrial and semi-arboreal and associated with at least six vegetation types from Caatinga and Atlantic Forest domains. None of the species is listed in threatened categories of IUCN, while two species are listed in the Brazilian Threatened Fauna list. Our data covers $32.34 \%$ of the state's area, a consequence of locally limited inventories and lack of longterm studies on snakes' fauna. The richest areas within the state are near large cities, which lack protected areas for the species they harbor, and highlights the necessity of protective policies and conservation actions.
\end{abstract}

Key words: Atlantic Forest, Caatinga, conservation, Serpentes, Squamata.

\section{INTRODUCTION}

Biodiversity is not equally distributed across Earth, varying significantly among and within geographic regions and taxa. The Neotropical region is one of the most biodiverse areas in the world (Olson et al. 2001, Antonelli \& Sanmartín 2011). Understanding the mechanisms and processes that generate and maintain the Neotropical hyper-diversity has been an important target in research and also a challenge since $19^{\text {th }}$ century naturalists began to study the region. Nowadays, this effort is still a central goal for researchers in systematic, ecology, biogeography, and evolutionary biology (Rull 2011, Hughes et al. 2013). One fundamental and practical limit of biodiversity knowledge is that scientists often work with incomplete and/or unrepresentative datasets. For instance, knowledge about the identity and distribution of species is biased (the Wallacean Shortfall), which compromises our capacity to synthesize knowledge of existing biodiversity, leading to misidentification of ecological and evolutionary processes and inefficient conservation strategies (Lomolino 2004, Hortal et al. 2015).

Squamate reptiles comprise the world's most diverse group of terrestrial vertebrates (Tonini et al. 2016, Uetz et al. 2020), and are arguably among the most neglected in conservation prioritization exercises (Schipper 
et al. 2008, Butchart \& Bird 2010, Böhm et al. 2013, Tingley et al. 2016). Within squamates, snakes comprise nearly 3,800 known species inhabiting temperate to tropical environments in varied habitats including terrestrial, marine, and freshwater areas (Wallach et al. 2014, Uetz et al. 2020). Snakes have a wide variety of ecological and morphological adaptations, and intriguing reproductive and dietary aspects and thus constitute an excellent system to investigate evolutionary processes (Cadle \& Greene 1993). However, the lack of knowledge about taxonomy and geographic distribution, for example, has hindered the inclusion of snakes in most largescale biodiversity and evolutionary studies (e.g. Pereira et al. 2010, Colston et al. 2013, Jenkins et al. 2015, Alencar et al. 2016, Card et al. 2016, Moura et al. 2016). Reliable estimates of the diversity and distribution of snakes would contribute to both global and regional understanding of evolutionary history of biotas and more accurate strategies for their conservation (Böhm et al. 2013, Meiri \& Chapple 2016).

In a recent study, Guedes et al. (2018) provided an overview of the distribution of Neotropical snakes based on a large database of georeferenced records representing 886 species and 12 families spanning 27 countries. To our knowledge, it is the most extensive and complete database of snake distribution for the Neotropical region. That study identified Amazonia, the Andes, and some portions of Northeastern Brazil as poorly sampled (less than 100 records per one-degree grid cell). As a consequence, these areas also present low richness (Guedes et al. 2018). The state of Rio Grande do Norte (RN hereafter) is located in northeastern Brazil and is also assumed to have low sampling and low species richness of snakes. Thus, a large knowledge gap identified in the literature for this state represents a serious shortcoming of present literature.
Many herpetofaunal studies in RN have focused on amphibians and lizards, while snake studies are por based on punctual observations and do not address local or state diversity. Indeed, some studies focused on snake association with bromeliads (Jorge et al. 2020), and chromatic anomaly (Paredero \& Passos 2020), while others reported endoparasites for two species (Almeida et al. 2008). In addition, accidents with venomous species and the regions where they occur have also been reported (Tavares et al. 2017, Costa et al. 2019). Otherwise, publications on the snakes of $\mathrm{RN}$ report single geographic distribution records (Brito P.S., unpublished data, Jorge \& Freire 2011, Brito \& Freire 2012, Santos et al. 2020, Aquino et al. 2020) or natural history notes (Souza \& Freire 2008, Sales et al. 2013, Ribeiro et al. 2014).

Snakes have also been part of local herpetofaunal inventories (Lima-Verde 1971, 1976, Sales et al. 2009, Caldas et al. 2016, Calixto \& Morato 2017, Coelho-Lima et al. 2020), and conservation inquiries such as how forest fragment characteristics (e.g., area, shape, isolation and matrix quality) affected reptiles in RN's Atlantic Forest (Lion et al. 2016). Finally, records of snakes for RN are mostly contained in broad natural history compilations (Schmidt \& Inger 1951, Guedes et al. 2014a, 2018) and taxonomic reviews (Thomas unpublished data, Myers \& Cadle 1994, Arredondo unpublished data, Fernandes \& Hamdan 2014, Pires et al. 2014, Montingelli et al. 2019). Costa \& Bérnils (2018) compiled the list of snake species for all Brazilian states based on presence and absence and listed for the first time a total of 43 species for RN without any further information.

Herein, we provide an updated list of snake species recorded inside the political limits of RN state through primary data, complemented by scientific literature. Additionally, for each species we present (1) the geographic distribution within 
the state, (2) information on habitat use, and (3) we discuss previous identification mistakes of species occurring in the state reported in the literature.

\section{MATERIALS AND METHODS}

\section{Study area}

The Northeast region of Brazil comprises a massive portion of the country with an area of 1,558,000 km² (IBGE 2017). Rio Grande do Norte state is the northeasternmost state in Brazil, between latitudes 4.82 and 6.98 South, and longitudes 38.58 and 34.96 West (Figure 1), and borders the states of Ceará and Paraíba. The state covers an area of $52,811 \mathrm{~km}^{2}$ corresponding to $0.62 \%$ of the Brazilian territory (Figure 1; IBGE 2017) and encompasses 167 municipalities arranged into four geographic mesoregions: Oeste Potiguar (62 municipalities), Central Potiguar (37 municipalities), Agreste Potiguar (43 municipalities), and Leste Potiguar (25 municipalities) (Figure 1; IDEMA 2008).

The natural landscapes in RN include a variety of geomorphological formations and phytophysiognomies. The relief includes plains and Tabuleiros along the coastal plain, lowland areas such as the sublitorânea and sertaneja

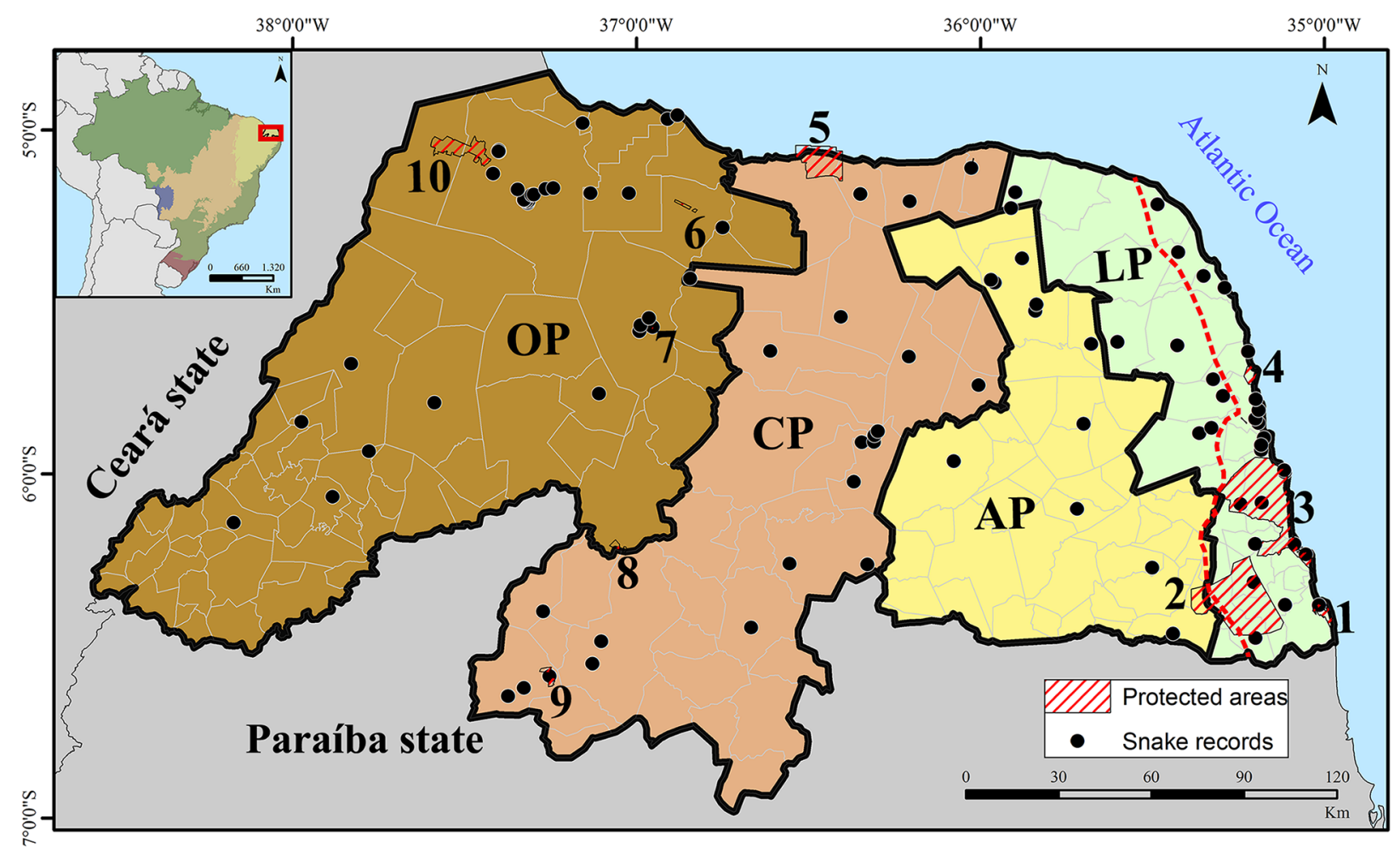

Figure 1. Map showing Rio Grande do Norte state and its four geographic mesoregions: Oeste Potiguar (OP), Central Potiguar (CP), Agreste Potiguar (AP), and Leste Potiguar (LP). The red dashed line shows the boundaries between Caatinga (left) and Atlantic Forest (right) biomes. Black dots represent snake records, hatched polygons show protected areas: RPPN Mata Estrela (private reserve) (1), APA Piriqui-Una protection area (2), APA Bonfim/Guaraíra protection area (3), APA Jenipabu (4), RDS Ponta do Tubarão (5), RPPN Esperança (6), FLONA Açu (7), RPPN Fazenda Salobro Farm (8), ESEC Seridó (9), PN Furna Feia (10). Protected area acronym, in increasing order of protection: APA (Área de Proteção Ambiental - Environmental Protection Area); RDS (Reserva de Desenvolvimento Sustentável - Sustainable Development Reserve); FLONA (Floresta Nacional - National Forest); RPPN (Reserva Particular do Patrimônio Natural -Private Reserve of the Natural Heritage); PN (Parque Nacional - National Park); ESEC (Estação Ecológica - Ecological Station). 
depressions, and mountain chains (e.g., Chapada da Serra Verde, Chapada do Apodi and Planalto da Borborema) (Diniz et al. 2015). The state is irrigated by sixteen river basins composed of seasonal rivers, lakes, and ponds. Two major river basins, Apodi-Mossoró and Piranhas-Assu, are responsible for the majority of the state's water reserves (Nascimento et al. 2014). The climate type according to Köppen-Geiger ranges from Tropical savanna (Aw) to Arid steppe (BSh) and desert hot (BWh) (Peel et al. 2007), with an average temperature of $28^{\circ} \mathrm{C}$. Precipitation is higher in the eastern coastal region $(1,100 \mathrm{~mm}$ per year) and decreases below $500 \mathrm{~mm}$ per year towards the west (Diniz \& Pereira 2015). Most of the state is within the Caatinga biome (95\% of the state area), while a narrow portion on the east coast is composed by Atlantic Forest (5\% of the state area) (Figure 1). Hence, the result is a vast vegetation mosaic composed of restinga sand dunes and mangroves (both with marine influence), seasonally dry formations such as caatinga bush, forest, and parkland vegetation, savanna-like fragments, and deciduous and semideciduous forests (IBGE 2012, SFB 2018).

\section{Data source}

We gathered snake distribution records by examining voucher specimens collected by us, housed in the collections of the Laboratorio de Anfíbios e Répteis at the Universidade Federal do Rio Grande do Norte (LAR-UFRN), and the Coleção Herpetológica do Semiárido at the Universidade Federal Rural do Semi-Árido (CHSA-UFERSA). Additionally, we compiled snake records from the scientific literature such as species inventories, ecological studies, taxonomic reviews, natural history and geographic distribution papers, dissertations, thesis, and books. We considered all records available from each paper and compared records with other studies to make sure we did not use the same record twice. We did not consider Lima-Verde $(1971,1976)$ whose records were not detailed for municipalities. We also included in our list photographic records and confirmation of records based on direct observation by the authors. We followed Burbrink et al. (2020) for family taxonomy.

We defined snake habitats based on records and the phytoecological units in RN state established by the national forest inventory (SFB 2018). When possible, we also provided information about habitat use from the location where the snake was collected or observed, complemented with literature information. We plotted snake records on a shapefile with federal and state protected areas to evaluate the degree of protection of each species in the state. We generated distribution maps for each species with habitat information using Mapbiomas 3.0 shapefiles. Finally, to evaluate distribution patterns of species richness, we compiled snake richness and geographic coordinates from all recorded municipalities and produced a Kernel density map restricted to RN with a search radius of 0.2 in Qgis 3.8.2.

\section{RESULTS}

We obtained 851 records (450 primary and 401 from literature) of 47 species from seven families (Dipsadidae, Colubridae, Elapidae, Boidae, Viperidae, Typhlopidae, and Leptotyphlopidae) and 33 genera. Dipsadidae and Colubridae were the most represented in species richness with 28 species (58.3\%) and 7 species (14.6\%), respectively. We recorded five species for the first time in RN: Apostolepis longicaudata, Bothrops leucurus, Dipsas mikanii, Imantodes cenchoa, and Thamnodynastes sertanejo. Most snakes in RN are terrestrial (66.7\%), followed by semiarboreal (25\%), fossorial (18.8\%), aquatic (16.7\%) and arboreal (10.4\%) species. Most 
species were found active during only one period of the day (40.4\% nocturnal, and $36.2 \%$ diurnal), while a small portion was found active on both periods (23.4\%) (Table I and Figures 2-5).

Our database contains at least one snake record in 54 (32.34\%) of the 167 municipalities in RN territory. When we consider the four mesoregions of the state, there were more records for municipalities in the Leste Potiguar (19 municipalities) with 277 snake occurrences, followed by Central Potiguar (14; 185), Oeste Potiguar (13; 322), and Agreste Potiguar (8; 67). The municipalities of Mossoró and Macaíba were the most representative in species richness (22 and 18 species, respectively), followed by Natal and Nísia Floresta (17 species each) (Figure 6A). The Kernel density map showed major richness on the Leste Potiguar and Mossoro regions (Oeste Potiguar), and additional scattered areas in João Câmara (Agreste Potiguar), Serra Negra do Norte (Central Potiguar), and Assú (Oeste Potiguar) (Figure 6B). Philodryas nattereri, Micrurus aff. ibiboboca, and Oxyrhopus trigeminus are widespread in RN and were recorded in 22, 16, and 15 municipalities, respectively (Figure 7). Detailed information of distribution maps for each species, sources, and snake species occurrences for all municipalities in $\mathrm{RN}$ are listed in the supporting information (Table SI and Figures S1-4).

Regarding the biomes, 660 records were from the Caatinga and 191 were from the Atlantic Forest. Twenty-two species were recorded in both biomes, while 13 species occurred only in Caatinga (Apostolepis longicaudata, Boiruna sertaneja, Crotalus durissus, Erythrolamprus miliaris, E. mossoroensis, Helicops angulatus, H. Leopardinus, Leptodeira annulata, Lygophis dilepis, Phimophis guerini, Sibon nebulatus, Thamnodynastes almae, and T. sertanejo) and 12 others only in Atlantic Forest (Amerotyphlops amoipira, A. paucisquamus, Bothrops leucurus, Drymarchon corais, Erythrolamprus almadensis, Hydrodynastes gigas, Imantodes cenchoa, Palusophis bifossatus, Micrurus cf. corallinus, M. potyguara, Psomophis joberti, and Spilotes pullatus). We detected snakes in six phytophisiognomies within RN. Snakes are predominantly reported for caatinga bush vegetation (37 species) and restinga sand dunes (29), followed by semideciduous forest (18), cerrado vegetation and caatinga forest vegetation (7 each), and caatinga parkland vegetation (5). Oxyrhopus trigeminus and Philodryas nattereri were detected in five vegetation types while other species were exclusive from restinga sand dunes (Amerotyphlops amoipira, Erythrolamprus almadensis, Bothrops leucurus, Micrurus potyguara, and Spilotes pullatus), caatinga bush vegetation (Micrurus aff. ibiboboca, Apostolepis longicaudata, Boiruna sertaneja, Erythrolamprus miliaris, E. mossoroensis, Helicops angulatus, H. leopardinus, Leptodeira annulata, Phimophis guerini, Thamnodynastes almae, and $T$. sertanejo), and semidecidual forest (Imantodes cenchoa and Psomophis joberti).

The IUCN lists Corallus hortulana, Bothrops erythromelas, Crotalus durissus, Erythrolamprus viridis, and Lygophis dilepis as Least Concern and Amerotyphlops amoipira as Data Deficient. The Red Book of Brazilian Threatened Fauna lists A. amoipira as endangered and A. paucisquamus as vulnerable (ICMBIO 2018). We recorded snake species inside the limits of three protected areas in the Leste Potiguar mesoregion (Environmental Protection Area of Apariqui-Una, EPA Bonfim/ Guanaíra, and Mata Estrela private reserve), one in the Central Potiguar mesoregion (Seridó Ecological Station), and one in the Oeste Potiguar mesoregion (Açu National Forest). Protected areas harbor 36 species, including three new records for RN, while Apostolepis longicaudata and Thamnodynastes sertanejo, both poorly known species (Curcio et al. 2011, Guedes et al. 2014a), were recorded only in unprotected areas. 
Table I. Snake species recorded in Rio Grande do Norte state, Brazil. The table outlines total records for each species (TR), total municipalities of occurrence (MO), biomes (Caatinga - CA and/or Atlantic Forest - AF), habitats (restinga sand dunes - RSD, caatinga bush vegetation - CBV, caatinga forest vegetation - CFV, caatinga parkland vegetation - CPV, cerrado - CE, and semideciduous forest - SDF. Snakes habits are as follows: aquatic (Aq), arboreal (Ar), fossorial (Fo), terrestrial (Te) and semiarboreal (SA). Activity period: diurnal (D) and nocturnal (N). $\left(^{*}\right)$ New state records.

\begin{tabular}{|c|c|c|c|c|c|c|c|c|c|c|c|}
\hline \multirow{2}{*}{ Species } & \multirow{2}{*}{ TR } & \multirow{2}{*}{ MO } & \multirow{2}{*}{ Biome } & \multicolumn{6}{|c|}{ Habitat } & \multirow{2}{*}{ Habit } & \multirow{2}{*}{ Activity } \\
\hline & & & & RSD & CBV & CFV & CPV & $\mathrm{CE}$ & SDF & & \\
\hline \multicolumn{12}{|l|}{ Leptotyphlopidae } \\
\hline $\begin{array}{l}\text { Epictia borapeliotes } \\
\text { (Vanzolini, 1996) }\end{array}$ & 32 & 7 & $C A, A F$ & $x$ & $x$ & & & & & Fo & N \\
\hline \multicolumn{12}{|l|}{ Typhlopidae } \\
\hline $\begin{array}{c}\text { Amerotyphlops } \\
\text { amoipira (Rodrigues \& } \\
\text { Juncá, 2002) }\end{array}$ & 2 & 1 & $A F$ & $x$ & & & & & & Fo & N \\
\hline $\begin{array}{c}\text { Amerotyphlops } \\
\text { paucisquamus (Dixon \& } \\
\text { Hendricks, 1979) }\end{array}$ & 6 & 4 & $A F$ & $x$ & & & & & $x$ & Fo & N \\
\hline \multicolumn{12}{|l|}{ Boidae } \\
\hline $\begin{array}{l}\text { Boa constrictor } \\
\text { Linnaeus, } 1758\end{array}$ & 22 & 7 & $\mathrm{CA}, \mathrm{AF}$ & $x$ & $x$ & & $x$ & & & Te, SA & $\mathrm{D}, \mathrm{N}$ \\
\hline $\begin{array}{l}\text { Corallus hortulana } \\
\text { (Linnaeus, 1758) }\end{array}$ & 6 & 3 & $\mathrm{CA}, \mathrm{AF}$ & & $x$ & & & & $x$ & $\operatorname{Ar}$ & N \\
\hline $\begin{array}{l}\text { Epicrates assisi } \\
\text { Machado, } 1945\end{array}$ & 14 & 9 & $\mathrm{CA}, \mathrm{AF}$ & $x$ & $x$ & $x$ & & & & Te, SA & N \\
\hline \multicolumn{12}{|l|}{ Viperidae } \\
\hline $\begin{array}{c}\text { Bothrops erythromelas } \\
\text { Amaral, } 1923\end{array}$ & 58 & 13 & $\mathrm{CA}, \mathrm{AF}$ & & $x$ & $x$ & & $x$ & & Te & N \\
\hline $\begin{array}{l}\text { Bothrops leucurus } \\
\text { Wagler in Spix } 1824^{*}\end{array}$ & 1 & 1 & $\mathrm{AF}$ & $x$ & & & & & & Te & N \\
\hline $\begin{array}{l}\text { Crotalus durissus } \\
\text { Wagler in Spix, } 1824\end{array}$ & 14 & 8 & CA & & $x$ & $x$ & & & & Te & N \\
\hline \multicolumn{12}{|l|}{ Elapidae } \\
\hline Micrurus aff. ibiboboca & 38 & 16 & $A F, C A$ & $x$ & $x$ & & & & $x$ & Fo & $\mathrm{D}, \mathrm{N}$ \\
\hline $\begin{array}{l}\text { Micrurus cf. corallinus } \\
\quad(\text { Merrem, 1820) }\end{array}$ & 2 & 2 & $A F$ & $x$ & & & & & $x$ & Fo & D \\
\hline $\begin{array}{c}\text { Micrurus potyguara } \\
\text { Pires, Da Silva Jr, } \\
\text { Feitosa, Prudente, } \\
\text { Pereira-Filho \& Zaher, } \\
2014\end{array}$ & 2 & 1 & AF & $x$ & & & & & & Fo & $\mathrm{D}, \mathrm{N}$ \\
\hline \multicolumn{12}{|l|}{ Colubridae } \\
\hline $\begin{array}{c}\text { Chironius flavolineatus } \\
\text { (Jan, 1863) }\end{array}$ & 28 & 6 & $C A, A F$ & $x$ & $x$ & & & $x$ & $x$ & SA & D \\
\hline $\begin{array}{l}\text { Drymarchon corais } \\
\text { (Boie, 1827) }\end{array}$ & 4 & 3 & $\mathrm{AF}$ & $x$ & $x$ & & & $x$ & & $\operatorname{Ar}$ & D \\
\hline
\end{tabular}


Table I. Continuation

\begin{tabular}{|c|c|c|c|c|c|c|c|c|c|c|c|}
\hline $\begin{array}{l}\text { Leptophis ahaetulla } \\
\text { (Linnaeus, 1758) }\end{array}$ & 15 & 8 & $C A, A F$ & $x$ & $x$ & $x$ & & & $x$ & $\mathrm{Ar}$ & D \\
\hline $\begin{array}{c}\text { Oxybelis aeneus } \\
\text { (Wagler in Spix, 1824) }\end{array}$ & 52 & 11 & $C A, A F$ & $x$ & $x$ & & $x$ & $x$ & & SA & D \\
\hline $\begin{array}{c}\text { Palusophis bifossatus } \\
\text { (Raddi, 1820) }\end{array}$ & 5 & 2 & $A F$ & $x$ & $x$ & & & & & $\mathrm{Te}$ & $D$ \\
\hline $\begin{array}{l}\text { Spilotes pullatus } \\
\text { (Linnaeus, 1758) }\end{array}$ & 3 & 2 & $A F$ & $x$ & & & & & & Te, SA & D \\
\hline $\begin{array}{l}\text { Tantilla melanocephala } \\
\text { (Linnaeus, 1758) }\end{array}$ & 16 & 8 & $C A, A F$ & $x$ & $x$ & & & & $x$ & Te, Fo & $\mathrm{D}, \mathrm{N}$ \\
\hline \multicolumn{12}{|l|}{ Dipsadidae } \\
\hline $\begin{array}{c}\text { Apostolepis cearensis } \\
\text { Gomes, } 1915\end{array}$ & 23 & 8 & $C A, A F$ & $X$ & $x$ & $x$ & & & $x$ & $\mathrm{Te}$ & $\mathrm{D}, \mathrm{N}$ \\
\hline $\begin{array}{c}\text { Apostolepis } \\
\text { longicaudata Gomes in } \\
\text { Amaral, 1921* }\end{array}$ & 1 & 1 & $C A$ & & $x$ & & & & & Te & $\mathrm{D}, \mathrm{N}$ \\
\hline $\begin{array}{l}\text { Boiruna sertaneja } \\
\quad \text { Zaher, } 1996\end{array}$ & 17 & 5 & CA & & $x$ & & & & & $\mathrm{Te}$ & N \\
\hline $\begin{array}{l}\text { Dipsas mikanii } \\
\text { (Schlegel, 1837)* }\end{array}$ & 4 & 3 & $C A, A F$ & $X$ & $x$ & & & & & Te & N \\
\hline $\begin{array}{c}\text { Erythrolamprus } \\
\text { almadensis (Wagler in } \\
\text { Spix, 1824) }\end{array}$ & 1 & 1 & $A F$ & $X$ & & & & & & Te & D \\
\hline $\begin{array}{l}\text { Erythrolamprus miliaris } \\
\quad(\text { Linnaeus, 1758) }\end{array}$ & 2 & 2 & $C A$ & & $x$ & & & & & $\mathrm{Te}, \mathrm{Aq}$ & $\mathrm{D}, \mathrm{N}$ \\
\hline $\begin{array}{c}\text { Erythrolamprus } \\
\text { mossoroensis (Hoge \& } \\
\text { Lima-Verde, 1973) }\end{array}$ & 7 & 4 & $C A$ & & $x$ & & & & & $\mathrm{Te}, \mathrm{Aq}$ & $\mathrm{D}, \mathrm{N}$ \\
\hline $\begin{array}{c}\text { Erythrolamprus } \\
\text { poecilogyrus (Wied, } \\
\text { 1825) }\end{array}$ & 28 & 9 & $C A, A F$ & $X$ & $x$ & & & & & $\mathrm{Te}, \mathrm{Aq}$ & $\mathrm{D}, \mathrm{N}$ \\
\hline $\begin{array}{c}\text { Erythrolamprus viridis } \\
\text { (Günther, 1862) }\end{array}$ & 47 & 6 & $C A, A F$ & $X$ & $x$ & & & & & $\mathrm{Te}, \mathrm{Aq}$ & D \\
\hline $\begin{array}{l}\text { Helicops angulatus } \\
\text { (Linnaeus, 1758) }\end{array}$ & 6 & 1 & CA & & $x$ & & & & & $\mathrm{Aq}$ & N \\
\hline $\begin{array}{l}\text { Helicops leopardinus } \\
\text { (Schlegel, 1837) }\end{array}$ & 1 & 1 & CA & & $x$ & & & & & $\mathrm{Aq}$ & N \\
\hline $\begin{array}{l}\text { Hydrodynastes gigas } \\
\text { (Duméril, Bibron \& } \\
\text { Duméril, 1854) }\end{array}$ & 3 & 3 & $A F$ & $X$ & & & & & $X$ & $\mathrm{Te}, \mathrm{Aq}$ & D \\
\hline $\begin{array}{l}\text { Imantodes cenchoa } \\
(\text { Linnaeus, 1758)* }\end{array}$ & 1 & 1 & $A F$ & & & & & & $x$ & $\mathrm{Ar}$ & N \\
\hline $\begin{array}{l}\text { Leptodeira annulata } \\
\quad \text { (Linnaeus, 1758) }\end{array}$ & 13 & 3 & CA & & $x$ & & & & & $\mathrm{Te}, \mathrm{SA}$ & N \\
\hline $\begin{array}{c}\text { Lygophis dilepis (Cope, } \\
\text { 1862) }\end{array}$ & 38 & 10 & $C A$ & & $x$ & & $x$ & & & $\mathrm{Te}, \mathrm{Aq}$ & D \\
\hline
\end{tabular}


Table I. Continuation

\begin{tabular}{|c|c|c|c|c|c|c|c|c|c|c|c|}
\hline $\begin{array}{l}\text { Oxyrhopus trigeminus } \\
\text { Duméril, Bibron \& } \\
\text { Duméril, } 1854\end{array}$ & 73 & 15 & $C A, A F$ & $x$ & $x$ & $x$ & & $x$ & $x$ & Te, SA & $\mathrm{D}, \mathrm{N}$ \\
\hline $\begin{array}{l}\text { Philodryas nattereri } \\
\text { Steindachner, } 1870\end{array}$ & 130 & 21 & $C A, A F$ & $x$ & $x$ & & $x$ & $x$ & $x$ & Te, SA & D \\
\hline $\begin{array}{l}\text { Philodryas olfersii } \\
\text { (Liechtenstein, 1823) }\end{array}$ & 43 & 12 & $C A, A F$ & $x$ & $x$ & $x$ & & & $x$ & $\mathrm{Te}, \mathrm{SA}$ & D \\
\hline $\begin{array}{c}\text { Philodryas } \\
\text { patagoniensis (Girard, } \\
\text { 1858) }\end{array}$ & 8 & 4 & $C A, A F$ & $x$ & $x$ & & & & $x$ & $\mathrm{Te}$ & D \\
\hline $\begin{array}{c}\text { Phimophis guerini } \\
\text { (Duméril, Bibron \& } \\
\text { Duméril, 1854) }\end{array}$ & 1 & 1 & CA & & $x$ & & & & & Te, Fo & $\mathrm{N}$ \\
\hline $\begin{array}{l}\text { Pseudoboa nigra } \\
\text { (Duméril, Bibron \& } \\
\text { Duméril, 1854) }\end{array}$ & 26 & 9 & $C A, A F$ & & $x$ & & $x$ & & & $\mathrm{Te}$ & $\mathrm{D}, \mathrm{N}$ \\
\hline $\begin{array}{l}\text { Psomophis joberti } \\
\text { (Sauvage, 1884) }\end{array}$ & 1 & 1 & $A F$ & & & & & & $x$ & Te, Fo & D \\
\hline $\begin{array}{l}\text { Sibon nebulatus } \\
\text { (Linnaeus, 1758) }\end{array}$ & 2 & 2 & CA & & $x$ & & & & $x$ & $\mathrm{Ar}$ & N \\
\hline $\begin{array}{c}\text { Taeniophallus } \\
\text { occipitalis (Jan, 1863) }\end{array}$ & 8 & 6 & $C A, A F$ & $x$ & $x$ & & & & $x$ & Te, Fo & D \\
\hline $\begin{array}{l}\text { Thamnodynastes almae } \\
\text { Franco \& Ferreira, } 2002\end{array}$ & 2 & 1 & $C A$ & & $x$ & & & & & Te, SA & $\mathrm{N}$ \\
\hline $\begin{array}{c}\text { Thamnodynastes } \\
\text { phoenix Franco, Trevine, } \\
\text { Montingelli \& Zaher, } \\
2017\end{array}$ & 28 & 9 & $C A, A F$ & $x$ & $x$ & & & $x$ & & Te, SA & $\mathrm{N}$ \\
\hline $\begin{array}{c}\text { Thamnodynastes } \\
\text { sertanejo Bailey, } \\
\text { Thomas \& Da Silva, } \\
\text { 2005* }\end{array}$ & 1 & 1 & $C A$ & & $x$ & & & & & $\mathrm{Te}, \mathrm{SA}$ & $\mathrm{N}$ \\
\hline $\begin{array}{l}\text { Xenodon merremii } \\
\text { (Wagler in Spix, 1824) }\end{array}$ & 16 & 5 & $C A, A F$ & $x$ & $x$ & & & & $x$ & $\mathrm{Te}$ & D \\
\hline
\end{tabular}



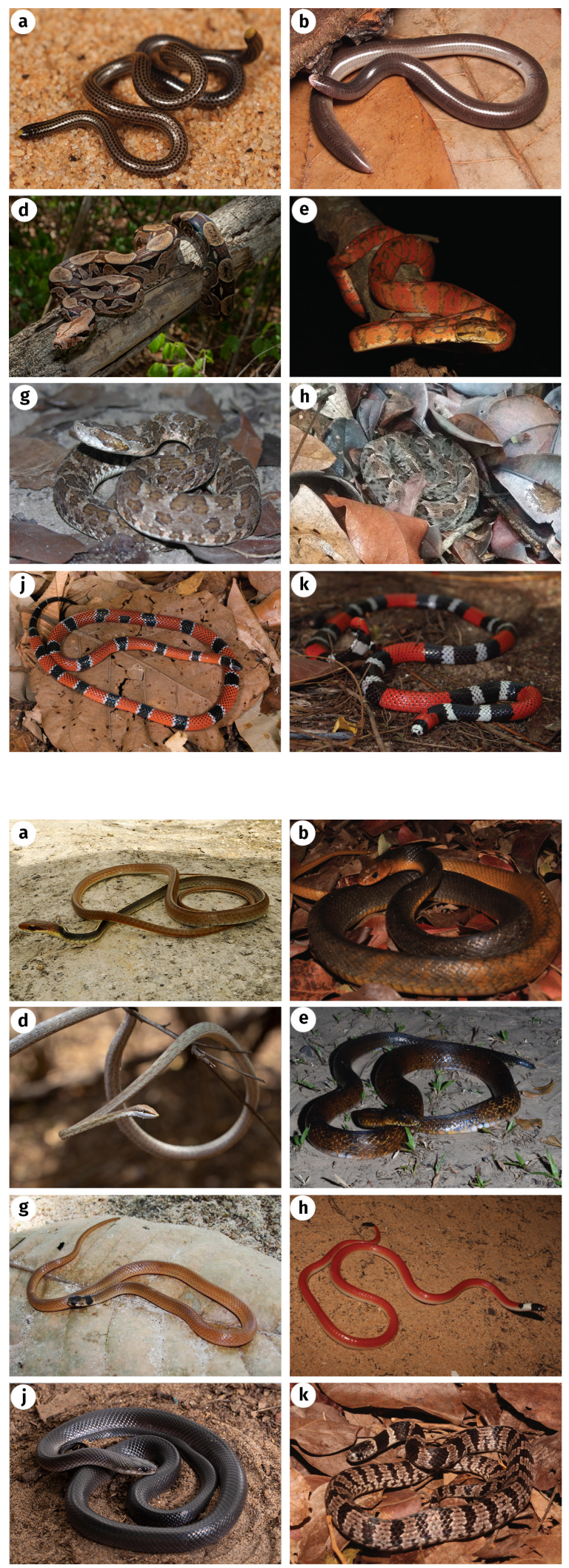
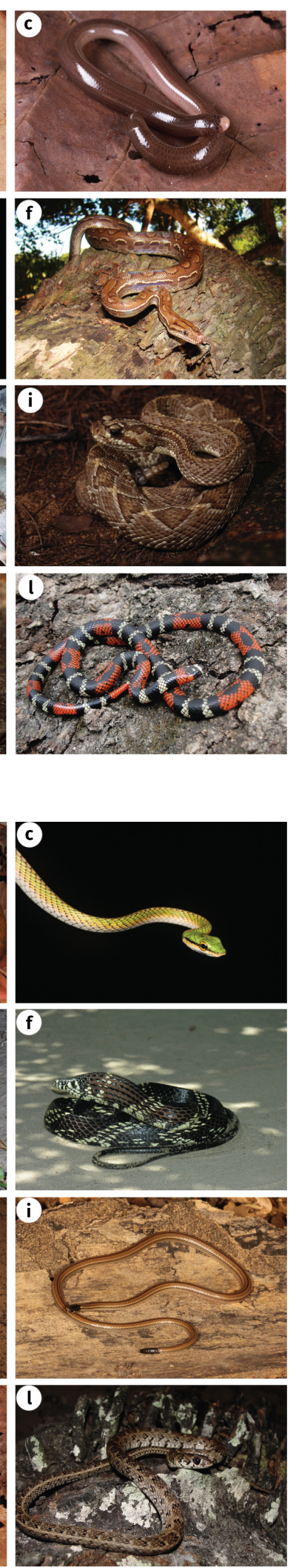

Figure 2. Snakes from Rio

Grande do Norte state, Brazil. Leptotyphlopidae: (a) Epictia borapeliotes. Typhlopidae: (b) Amerotyphlops amoipira, (c) A. paucisquamus. Boidae: (d) Boa constrictor, (e) Corallus hortulana, (f) Epicrates assisi. Viperidae: (g) Bothrops erythromelas, (h) Bothrops leucurus, (i) Crotalus durissus. Elapidae: (j) Micrurus cf. corallinus, (k) M. aff. ibiboboca, and (l) M. potyguara. Photo $b$ by D.J. Santana and photo $h$ by J.L.G.S. Silveira.

Figure 3. Snakes from Rio Grande do Norte state, Brazil. Colubridae: (a) Chironius flavolineatus, (b) Drymarchon corais, (c) Leptophis ahaetulla, (d) Oxybelis aeneus, (e) Palusophis bifossatus, (f) Spilotes pullatus, (g) Tantilla melanocephala. Dipsadidae: (h) Apostolepis cearensis, (i) A. longicaudata, (j) Boiruna sertaneja, (k) Dipsas mikanii, and (l) Erythrolamprus almadensis. Photo e by D.J. Santana. 

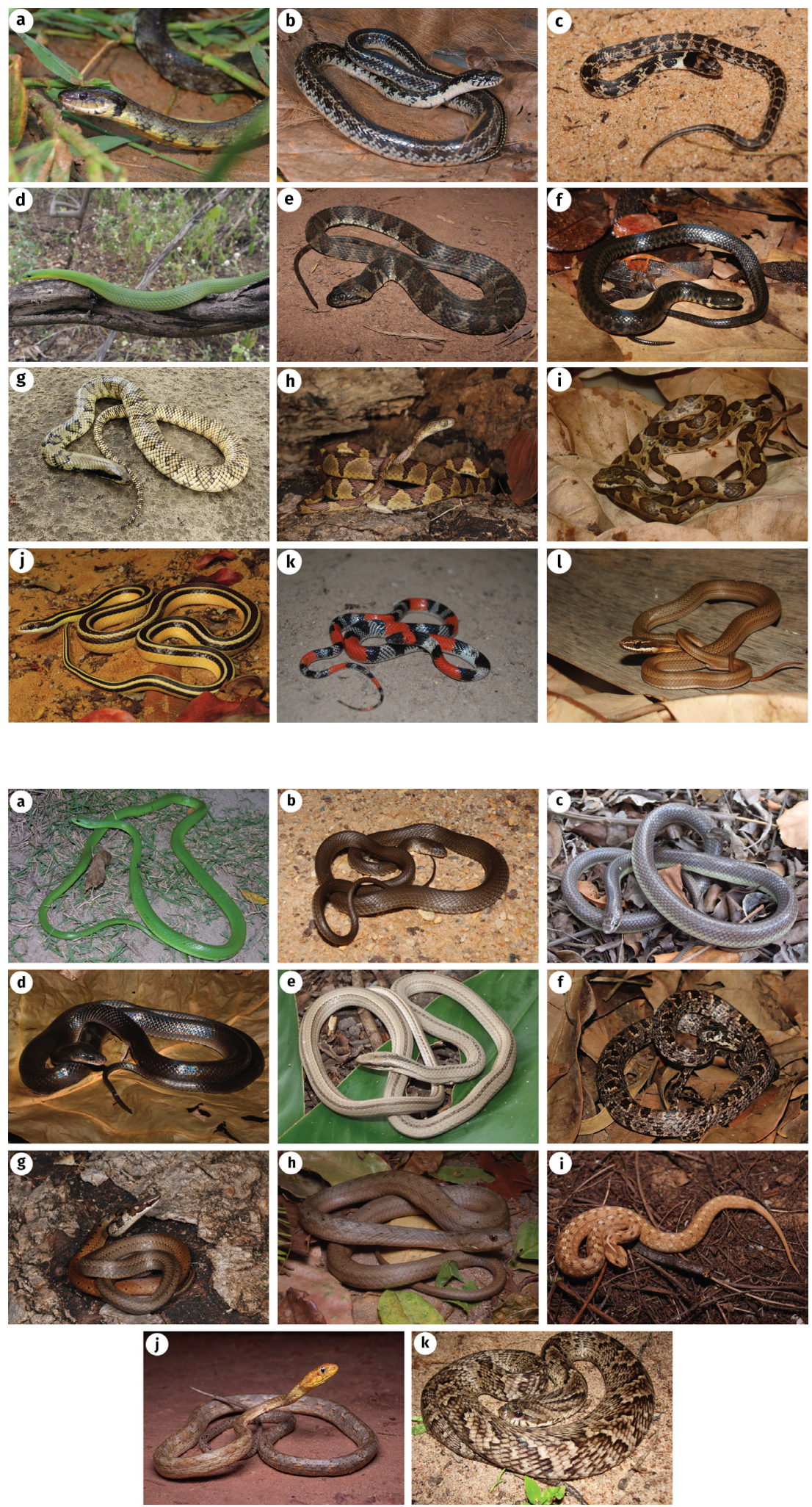

Figure 4. Snakes from Rio Grande do Norte state, Brazil. Dipsadidae: (a) Erythrolamprus miliaris, (b) E. mossoroensis, (c) E. poecilogyrus, (d) E. viridis, (e) Helicops angulatus, (f) H. leopardinus, (g) Hydrodynastes gigas, (h) Imantodes cenchoa, (i) Leptodeira annulata, (j) Lygophis dilepis, (k) Oxyrhopus trigeminus, ( $(\mathbf{)}$ Philodryas nattereri. Photo d by B. Pontes.

Figure 5. Snakes from Rio Grande do Norte state, Brazil. Dipsadidae: (a) Philodryas olfersii, (b) P. patagoniensis, (c) Phimophis guerini, (d) Pseudoboa nigra, (e) Psomophis joberti, (f) Sibon nebulatus, (g) Taeniophallus occipitalis, (h) Thamnodynastes almae, (i) T. phoenix, (j) T. sertanejo, and (k) Xenodon merremii. Photos $\mathrm{C}$ and e by G.A. Pereira-Filho. 


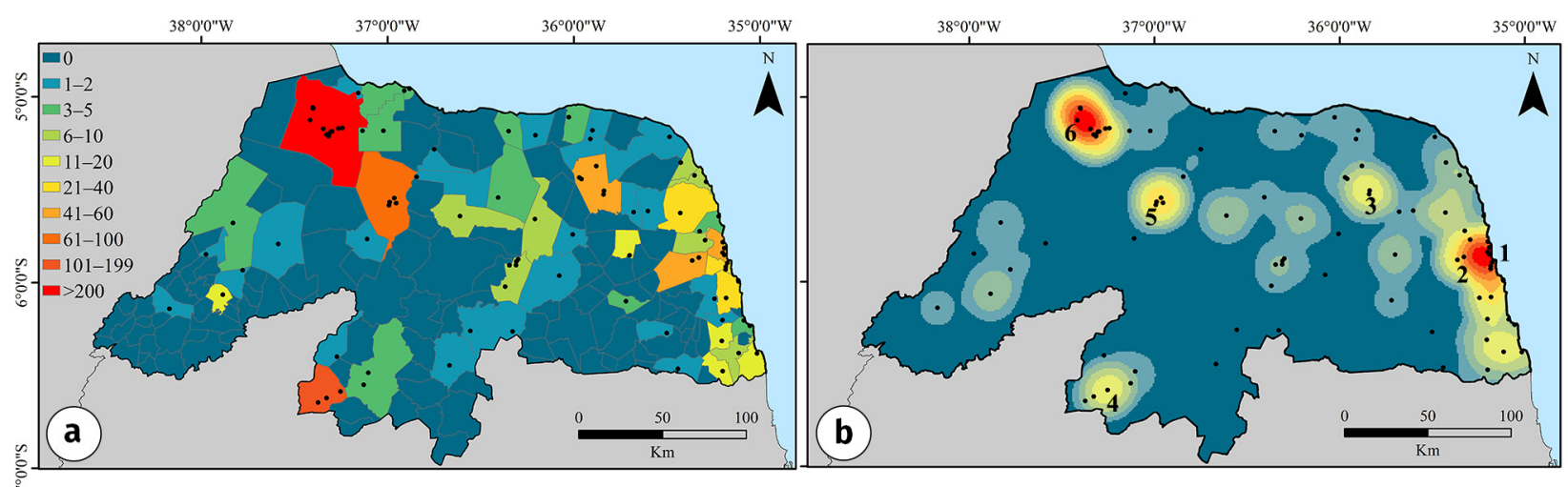

Figure 6. Frequency of snake records in municipalities of Rio Grande do Norte (RN) state, Brazil (a) and Kernel density map showing the concentration of snake richness over RN (b). Warmer colors show higher richness and frequency, black dots represent snake records. We highlight the municipalities of Natal (1), Macaíba (2), João Câmara (3), Serra Negra do Norte (4), Assú (5), and Mossoró (6).

\section{DISCUSSION}

\section{Snake richness and habitat use overview in Rio Grande do Norte}

This is the largest compilation of snake records for Rio Grande do Norte to date, and we generate detailed maps of snake occurrences and patterns of species richness, along with quantifying sampling gaps. We also report new occurrences, geographic distribution extensions, and habitat information for all species. The species richness of RN is somewhat low when compared to other state compilations for Brazil, such as Bahia (131 spp.; Curcio et al. 2012, Hamdan \& Lira-da-Silva 2012, Fernandes \& Hamdan 2014), Rondônia (119 spp.; Bernarde et al. 2012, Passos et al. 2016), Mato Grosso do Sul (113 spp.; Ferreira et al. 2017), Ceará (67 spp.; Roberto \& Loebmann 2016, Borjes-Nojosa et al. 2017), and Paraíba (63 spp.; Pereira-Filho et al. 2017).

Many factors can affect species richness, including diversity of habitats, area, and sampling effort (Bracewell et al. 2018). For example, Bahia and Mato Grosso do Sul states, while also being much larger than RN, harbor three distinct biomes of forested and open formations. Ceará state is also larger than RN, and although the Atlantic Forest does not reach the state, it harbors many relictual enclaves of humid forests on top of high-altitude areas maintained by orographic rainfall. Larger area and environmental complexity allow higher richness and support for endemic species to persist over time (Roberto \& Loebmann 2016). Aside from environmental features, areas with high species richness are usually better sampled at local level (Guedes et al. 2018). Indeed, the low snake species richness in RN is very likely a sampling artifact resulting from the reduced number of studies for the group in RN compared to the neighboring states of Ceará and Paraíba (Santana et al. 2008, Loebmann \& Haddad 2010, Mesquita et al. 2011, 2012, 2013, Pereira-Filho \& Montingelli, 2011, França et al. 2012, Roberto \& Loebmann, 2016, Costa et al. 2018, Sampaio et al. 2018).

Some species reported herein are known from single or old records for RN (e.g. Psomophis joberti, Erythrolamprus almadensis, Helicops leopardinus, $H$. angulatus). Most of these species are frequent in other regions (Guedes et al. 2014a, Marques et al. 2016, Mesquita et al. 2018, França et al. 2020) and the lack of recent records in $\mathrm{RN}$ reinforces the scarcity of snake studies in the state. Likewise, the five new species recorded for the state further suggests that the 


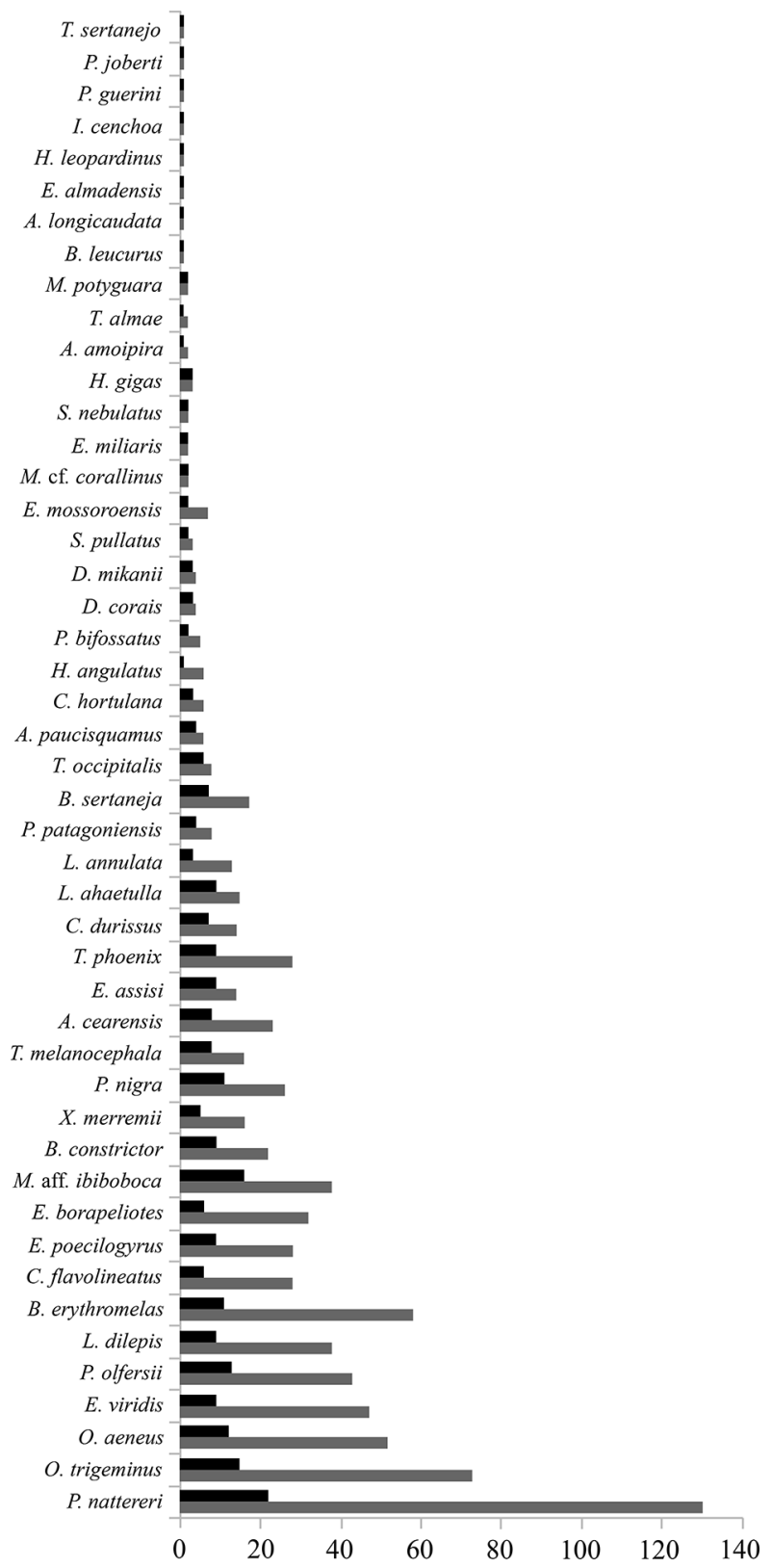

Figure 7. Total records for all snake species (gray bars) and total municipalities where they were recorded (black bars) in Rio Grande do Norte state, Brazil.

state is still heavily understudied. Knowledge of snake species richness is concentrated in areas easily accessed by researchers, leading to a lack of snake records on most of the state's territory, hindering our ability to correctly assess the overall diversity and conservation status of these animals in the state (Meyer et al. 2015, Oliveira et al. 2016).
Over $65 \%$ of the localities we list and $29 \%$ of the state's area have three or fewer species registered. Furthermore, areas with the highest species richness are within/near the municipalities of two of the largest cities (Natal and Mossoró) or inside protected areas with research infrastructure (Açu National Forest and Seridó Ecological Station). Such correlation of sampling intensity near larger cities and research facilities is expected (Meyer et al. 2015, Oliveira et al. 2016).

Most species were recorded in Caatinga bush vegetation, which covers $84 \%$ of RN state (SFB 2018). Although restinga sand dune habitats cover around $4 \%$ of $\mathrm{RN}$ vegetation, this habitat is distributed along the seashore of RN where densely populated cities are found. Despite the sampling artifact, snake richness is high throughout Atlantic Forest habitats (Argôlo 2004, Santana et al. 2008, Pereira-Filho \& Montingelli 2011, Marques et al. 2016, Guedes et al. 2018, Sampaio et al. 2018). This raises concerns, as the biome is threatened by ongoing anthropogenic actions and habitat loss (Joly et al. 2014) and is mostly restricted to small fragments embedded in sugar cane plantations in RN (Lion et al. 2016). Even though the Atlantic Forest covers a small portion of RN state, it showed similar richness to the Caatinga biome, reinforcing the conservation importance of this biome and its related ecosystems for biodiversity. Some species recorded in restinga sand dunes (e.g. Erythrolamprus almadensis, Bothrops leucurus, and Spilotes pullatus) are also common in this habitat in other regions (Miranda et al. 2012, Marques et al. 2016). Oxyrhopus trigeminus and Philodryas nattereri are generalist species and inhabit many vegetation types along their distributions (Pereira-Filho \& Montinguelli 2011, Guedes et al. 2014a, Marques et al. 2016), and were also recorded in almost all habitats in RN. All species exclusive to caatinga bush vegetation 
are typical from open habitat formations and likely occur in this habitat (Guedes et al. 2014a). Likewise, Imantodes cenchoa and Psomophis joberti were only found in semideciduous forest, as the former is an arboreal species typical from forests and the later occurs in many habitats from Caatinga, Cerrado, and Atlantic Forest (Moura et al. 2013, Marques et al. 2016, Mesquita et al. 2018).

\section{Geographic distribution records and taxonomic accounts}

Our data added five new species records for RN state. Apostolepis longicaudata is mostly reported for areas of cerrado vegetation (Curcio et al. 2011) and here it was recorded in João Câmara municipality (5.37346 S, 35.879398 W, WGS84, $188 \mathrm{~m}$ a.s.l.) where xeric vegetation prevails. This record extends its distribution to 237 km North of Cabaceiras, Paraíba state (Curcio et al. 2011, Pereira-Filho et al. 2017) and 1281 km East from Estreito, Maranhão state (França et al. 2018). Bothrops leucurus inhabits mainly forests (Argôlo 2004) and the nearest record is from REBIO Guaribas (Reserva Biológica, or Biological Reserve, a strict protection area in Brazil), between the municipalities of Rio Tinto and Mamanguape, Paraíba state (Mesquita et al. 2018), $38 \mathrm{~km}$ south from our record in Baía Formosa (6.381705 S, $35.016233 \mathrm{~W}, 63 \mathrm{~m}$ asl). We recorded Dipsas mikanii in Macaíba (5.866257 S, 35.32931 W, 42 m a.s.l.), Nísia Floresta (6.083561 S, 35.18251 W, $59 \mathrm{~m}$ a.s.l.), and Parnamirim (5.915981 S, 35.18419 W, $34 \mathrm{~m}$ a.s.l.). These records are $466 \mathrm{~km}$ Northeast of Barbalha, Ceará state (Roberto and Loebmann 2016) and 72 km North of Mamanguape, in Paraíba state (Mesquita et al. 2018). We also recorded Imantodes cenchoa in Baía Formosa, 38 km northward from REBIO Guaribas in Paraíba state (Mesquita et al. 2018) and $495 \mathrm{~km}$ eastward from the municipality of Crato, Ceará state (Roberto and Loebmann,
2016). Finally, Thamnodynastes sertanejo is endemic from Caatinga, and our record in João Câmara (5.526461 S, 35.841056 W, 160 m a.s.l.) extends its distribution $423 \mathrm{~km}$ Northeast of Jati, Ceará state, and $320 \mathrm{~km}$ Northeast of Sertânia, Pernambuco state (Coelho et al. 2013).

Previous records within $\mathrm{RN}$ require clarification. Sales et al. (2009) reported Epicrates cenchria for sand dunes in the municipality of Natal without specifying its subspecies. Passos and Fernandes (2008) reviewed and elevated the subspecies of Epicrates to species. Epicrates cenchria occurs in Amazonia and Atlantic Forest in primary, ombrophilous, and disturbed forests (Martins \& Oliveira 1998, Argôlo 2004). Though the municipality of Natal is within the Atlantic Forest biome, its vegetation is strongly influenced by Caatinga and this record actually corresponds to E. assisi (R. Sales pers. comm.). Large patches of semideciduous forests are abundant on the southeast portion of the state, near the Paraíba state boundary, where we recorded typical Atlantic Forest species as Corallus hortulana and Bothrops leucurus. Schmidt \& Inger (1951) and Freire et al. (2009) reported the occurrence of Lygophis lineatus but this species was restricted to Amazonia after review by Hoge (1952), while L. dilepis has a broad distribution in RN state, as already pointed by Guedes et al. (2014a).

The compilation of Costa \& Bérnils (2018) reported Micrurus lemniscatus carvalhoi, Amerotyphlops brongersmianus and Erythrolamprus viridis viridis for RN. Micrurus lemniscatus carvalhoi was recorded by Schmidt \& Inger (1951) in Nísia Floresta, but later review by Pires et al. (2014), who showed this to be a novel species, M. potyguara. As for A. brongersmianus, Lion et al. (2016) recorded it in Goianinha, but we examined the voucher specimen and scale counts (18 rows around the body and 214 middorsal scales) are typical of A. paucisquamus (Hedges et al. 2014). Finally, 
Sales et al. (2009) reported E. viridis for the sand dunes in Natal and Costa \& Bérnils (2018) considered the subspecies E. v. viridis because the record was from the Atlantic Forest biome ( $\mathrm{H}$. Costa pers. comm.). We believe this situation is similar to that of Epicrates cenchria/E. assisi, and the subspecies that must be considered for the state is Erythrolamprus viridis prasinus. As we did not examine its voucher, however, further investigation is needed to clarify this issue. Lastly, species like E. almadensis (Guedes et al. 2014a) and Spilotes pullatus (Sales et al. 2009) are reported here but are absent in the compilation of Costa \& Bérnils (2018).

The holotype of Micrurus ibiboboca was described from the Atlantic Forest (Belmonte, Bahia state) with a diagnosis of 210 ventral scales and 23 subcaudal scales (Merrem 1820). Vanzolini et al. (1980) reported that specimens from the Caatinga were not properly described in the literature, since they obtained snakes with 219-243 ventral scales. Then, Argôlo (2004) examined specimens of $M$. ibiboboca from Southeast Bahia with 192-219 ventral scales and 16-26 subcaudal scales, which matched the holotype of $M$. ibiboboca. The segregation between specimens from Caatinga and Atlantic Forest was also highlighted in Guedes et al. (2014a), but Pires et al. (2014) treated them as a single entity. Ventral scales from specimens of RN ranged from 226 to 250 and subcaudal scales from 24 to 30 without proper segregation between both biomes. Until a more detailed taxonomic review of these species is available, we propose specimens from RN should be treated as Micrurus aff. ibiboboca.

\section{Conservation challenges for Rio Grande do Norte snakes}

The biggest conservation challenge for snake species in RN in face of rising habitat loss is to identify priority areas for conservation considering current sampling gaps. RN still holds $42 \%$ of its original vegetation coverage (SFB 2018). Though $6.2 \%$ of RN's area corresponds to protected areas (SFB 2018, IDEMA 2019), most of these confer low protection (Environmental Protection Areas, or APAs) and just a small part of the Caatinga $\left(55.37 \mathrm{~km}^{2}\right)$ that occurs in the state is protected by two Strict Protection areas (SPA), Seridó Ecological Station and Furna Feia National Park.

Most snake species recorded for RN show broad distributions, occurring in other biomes and neighboring states (Guedes et al. 2014a). Additionally, most of them are also recorded in SPA along their distribution and also in RN (Guedes et al. 2014b, this work). Because of this, only two species (Amerotyphlops amoipira and A. pausicquamus) are listed by ICMBio in threatened categories for the State, while none are listed in IUCN (ICMBio 2018, IUCN 2019). Nevertheless, evidence suggests that some species should be monitored carefully in future assessments. The coral snake Micrurus potyguara was recently described for a small area of the Atlantic Forest of RN, Paraíba, and Pernambuco states (Pires et al. 2014). Its habitat is reported to be declining, which might lead the species to be listed in IUCN categories in the future, as has occurred with other squamate species in similar conditions recently (Fazolato et al. 2017, Rosário et al. 2019). Erythrolamprus mossoroensis is a Caatinga endemic that occurs in four municipalities of RN; although this species has a large range (Guedes et al. 2014a), most records are old, possibly indicating a lowdensity population or lack of faunal inventories. Similarly, Boiruna sertaneja is considered rare in the neighboring state of Paraíba (PereiraFilho et al. 2017) and might be threatened by intrinsic aspects of the species (Pizzatto 2005), besides anthropogenic factors. It is urgent for RN state to evaluate the conservation status of 
its flora and fauna, including snakes, and draft lists of endangered species to guide political and conservation actions aiming to maintain the remaining habitats.

Our data updates the current knowledge of snakes in RN and the necessity for a significant increase in sampling effort within many poorly sampled regions of the state. The low number of records compared to other regions is likely the result of limited local inventories and fewer long-term studies on the snake fauna. Several undersampled regions might gather similar richness to those observed in the East region of RN, and even produce new state records or undescribed species. Finally, those areas reported herein as the richest in snake species in RN require protective policies and conservation actions for the species they harbor.

\section{Acknowledgments}

We thank Bruna Pontes, Diego J. Santana, Gentil A. PereiraFilho, and João L.A. Silveira for kindly providing snake photographs. We also thank Alex Pyron for the English review. RM thanks Coordenação de Aperfeiçoamento de Pessoal de Nivel Superior for the current doctoral fellowship (CAPES Process number 1489596). TBG thanks to Universidade Estadual do Maranhão for the Senior Researcher fellowship. AAG thanks Conselho Nacional de Desenvolvimento Científico e Tecnológico (CNPq) for his productivity research grant (\#310942/2018-7).

\section{REFERENCES}

ALENCAR LRV, QUENTAL TB, GRAZZIOTIN FG, ALFARO ML, MARTINS M, VENZON M \& ZAHER H. 2016. Diversification in vipers: Phylogenetic relationships, time of divergence and shifts in speciation rates. Mol Phylogenet Evol 105: 50-62.

ALMEIDA WO, GUEDES TB, FREIRE EMX \& VASCONCELLOS A. 2008. Pentastomid infection in Philodryas nattereri Steindachner, 1870 and Oxybelis aeneus (Wagler, 1824) (Squamata: Colubridae) in a caatinga of northeastern Brazil. Braz J Bio 68: 193-197.

ANTONELLI A \& SANMARTÍN I. 2011. Why are there so many plant species in the Neotropics? Taxon 60: 403-414.
AQUINO NC, MAIA HAC, JUCÁ MAS \& PASSOS DC. 2020. Distribution extension and update map of Taeniophallus occipitalis (Jan 1863)(Squamata, Dipsadidae), with a relevant record to Caatinga, Northeast Brazil. Herp Notes 13: 661-666.

ARGÔLO AJS. 2004. As Serpentes dos Cacauais do Sudeste da Bahia. Ilhéus: Editus, 251 p.

BERNARDE PS, ALBUQUERQUE S, BARROS TO \& TURCI LCB. 2012. Serpentes do Estado de Rondônia, Brasil. Biota Neotrop 12: v154-182.

BÖHM ET AL. 2013. The conservation status of the world's reptiles. Biol Conserv 157: 372-385.

BORJES-NOJOSA DM, LIMA DC, BEZERRA CH \& HARRIS DJ. 2017. Two new species of Apostolepis Cope, 1862 (Serpentes: Elapomorphini) from brejos de altitude in Northeastern Brazil. Rev Nordest Zool 10: 74-94.

BRACEWELL SA, CLARK GF \& JOHNSTON EL. 2018. Habitat complexity effects on diversity and abundance differ with latitude: an experimental study over 20 degrees. Ecology 99: 1964-1974.

BRITO PS \& FREIRE EMX. 2012. New records and geographic distribution map of Typhlops amoipira Rodrigues and Juncá, 2002 (Typhlopidae) in the Brazilian Rainforest. Check List 8: 1347.

BURBRINK ET AL. 2020. Interrogating Genomic-Scale data for Squamata (Lizards, Snakes, and Amphisbaenians) shows no support for key traditional morphological relationships. Syst Biol 69: 502-520.

BUTCHART SHM \& BIRD JP. 2010. Data Deficient birds on the IUCN Red List: What don't we know and why does it matter? Biol Conserv 143: 239-247.

CADLE JE \& GREENE HW. 1993. Phylogenetic patterns, biogeography, and the ecological structure of Neotropical snake assemblages. In: Ricklefs RE \& Schluter D (Eds), Species Diversity In Ecological Communities, Chicago: University of Chicago Press, Chicago, USA, p. 281-293.

CALDAS FLS, COSTA TB, LARANJEIRAS DO, MESQUITA DO \& GARDA AA. 2016. Herpetofauna of protected areas in the Caatinga V: Seridó Ecological Station (Rio Grande do Norte, Brazil). Check List 12: 1929.

CALIXTO PO \& MORATO SAA. 2017. Herpetofauna recorded by a fauna rescue program in a Caatinga area of João Câmara, Rio Grande do Norte, Brazil. Check List 13: 647-657.

CARD et al. 2016. Phylogeographic and population genetic analyses reveal multiple species of Boa and independent 
origins of insular dwarfism. Mol Phylogenet Evol 102: 104-116.

COELHO RDF, SOUZA K, WEIDER AG, PEREIRA LCM \& RIBEIRO LB. 2013. Overview of the distribution of snakes of the genus Thamnodynastes (Dipsadidae) in northeastern Brazil, with new records and remarks on their morphometry and pholidosis. Herpetol Notes 6: 355-360.

COELHO-LIMA AD, JUCÁ MAS, FONSECA EBF, MEDEIROS LCV, SOARES PBC, CUNHA PVA \& PASSOS DC. 2020. Rediscovering a forgotten scientific collection in the Rio Grande do Norte State, Brazil: The herpetological collection of the zoologist José Santiago Lima-Verde. Biota Neotrop 20(1): e20180706.

COLSTON TJ ET AL. 2013. Molecular systematics and historical biogeography of tree boas (Corallus spp.). Mol Phylogenet Evol 66: 953-959.

COSTA HC \& BÉRNILS RS. 2018. Répteis do Brasil e suas Unidades Federativas: Lista de espécies. Herp Brasil 7: 11-57.

COSTA MKB, FONSECA CS, NAVONI JA \& FREIRE EMX. 2019. Snakebite accidents in Rio Grande do Norte state, Brazil: Epidemiology, health management and influence of the environmental scenario. Trop Med \& Int Health 24: 432-441.

COSTA ET AL. 2018. Herpetofauna of protected areas in the Caatinga VII: Aiuaba Ecological Station (Ceará, Brazil). Herpetol Notes 11: 929-941.

CURCIO FF, NUNES PMS, ARGÔLO AJS, SKUK G \& RODRIGUES MT. 2012. Taxonomy of the South American Dwarf Boas of the genus Tropidophis Bibron, 1840, with the description of two new species from the Atlantic Forest (Serpentes: Tropidophiidae). Herpetol Monogr 26: 80-121.

CURCIO FF, NUNES PMS, HARVEY MB \& RODRIGUES MT. 2011. Redescription of Apostolepis longicaudata (Serpentes: Xenodontinae) with comments on its hemipenial morphology and natural history. Herpetologica 67: 318-331.

DINIZ MTM \& PEREIRA VHC. 2015. Climatologia do estado do Rio Grande do Norte, Brasil: Sistemas atmosféricos atuantes e mapeamento de tipos de clima. Bol Goiano Geog 35: 488-506.

FAZOLATO C, FERNANDES F \& BATALHA-FILHO H. 2017. The effects of Quaternary sea-level fluctuations on the evolutionary history of an endemic ground lizard (Tropidurus hygomi). Zool Anz 270: 1-8.

FERNANDES DS \& HAMDAN B. 2014. A new species of Chironius Fitzinger, 1826 from the state of Bahia, Northeastern Brazil (Serpentes: Colubridae). Zootaxa 3881: 563.
FERREIRA ET AL. 2017. Répteis do Mato Grosso do Sul, Brasil. Iheringia Sér Zool 107: 1-13.

FRANÇA DPF, BARBO FE, SILVA-JR NJ, SILVA HLR \& ZAHER H. 2018. A new species of Apostolepis (Serpentes, Dipsadidae, Elapomorphini) from the Cerrado of Central Brazil. Zootaxa 4521: 539-552.

FRANÇA RC, GERMANO CES \& FRANCA FGR. 2012. Composition of a snake assemblage inhabiting an urbanized area in the Atlantic Forest of Paraíba state, Northeast Brazil. Biota Neotrop 12: 183-195.

FRANÇA RC, MORAIS M, FRANÇA FGR, RÖDDER D, SOLÉ M. 2020. Snakes of the Pernambuco Endemism Center, Brazil: diversity, natural history and conservation. Zookeys 1002: 115-158.

FREIRE EMX, SKUK GO, KOLODIUK MF, RIBEIRO LB, MAGGI BS, RODRIGUES LS, VIEIRA WLS \& FALCÃO ACGP. 2009. Répteis Squamata das Caatingas do Seridó do Rio Grande do Norte e do Cariri da Paraíba: síntese do conhecimento atual e perspectivas, p. 51-84, In: Freire EMX (Eds), Recursos naturais das Caatingas: uma visão multidisciplinar. Natal: Editora da UFRN.

GUEDES TB, NOGUEIRA CC \& MARQUES OAV. 2014a. Diversity, natural history, and geographic distribution of snakes in the Caatinga, Northeastern Brazil. Zootaxa 3863: 1-93.

GUEDES TB, SAWAYA RJ \& NOGUEIRA CC. 2014b. Biogeography, vicariance and conservation of snakes of the neglected and endangered Caatinga region, north-eastern Brazil J Biogeogr 41: 919-931.

GUEDES ET AL. 2018. Patterns, biases and prospects in the distribution and diversity of Neotropical snakes. Global Ecol Biogeogr 27: 14-21.

HAMDAN B \& LIRA-DA-SILVA RM. 2012. The snakes of Bahia state, northeastern Brazil: Species richness, composition and biogeographical notes. Salamandra 48: 31-50.

HEDGES SB, MARION AB, LIPP KM, MARIN J \& VIDAL N. 2014. A taxonomic framework for typhlopid snakes from the Caribbean and other regions (Reptilia, Squamata). Carib Herpetol 49: 1-61.

HOGE AR. 1952. Notes on Lygophis Fitzinger with revalidation of two subspecies. Mem. I. Butantan 24: 245-268.

HORTAL J, BELLO F, DINIZ-FILHO JAF, LEWINSOHN TM, LOBO JM \& LADLE RJ. 2015. Seven shortfalls that beset largescale knowledge of biodiversity. Ann Rev Ecol Evol S 46: 523-549. 
HUGHES CE, PENNINGTON RT \& ANTONELLI A. 2013. Neotropical plant evolution: Assembling the big picture. Bot J Linn Soc 171: 1-18.

IBGE - INSTITUTO BRASILEIRO DE GEOGRAFIA E ESTATÍSTICA. 2012. Manual técnico da vegetação brasileira. IBGE, Rio de Janeiro.

IBGE - INSTITUTO BRASILEIRO DE GEOGRAFIA E ESTATÍSTICA. 2017. Cidades e Estados, https://www.ibge.gov.br/cidades-eestados/rn.html?, accessed in $4^{\text {th }}$ March 2019.

ICMBIO - INSTITUTO CHICO MENDES DE CONSERVAÇÃO DA BIODIVERSIDADE. 2018. Livro Vermelho da fauna brasileira ameaçada de extinção. ICMBio, Brasília, Distrito Federal.

IDEMA - INSTITUTO DE DESENVOLVIMENTO ECONÔMICO E MEIO AMBIENTE DO RIO GRANDE DO NORTE. 2008. Perfil do seu Município, http://www.idema.rn.gov.br, accessed $15^{\text {th }}$ May 2019.

IDEMA - INSTITUTO DE DESENVOLVIMENTO ECONÔMICO E MEIO AMBIENTE DO RIO GRANDE DO NORTE. 2019. Unidades de conservação, http://www.idema.rn.gov.br, accessed $15^{\text {th }}$ May 2019.

IUCN - INTERNATIONAL UNION FOR CONSERVATION OF NATURE. 2019. The IUCN Red List of Threatened Species, http:// www.iucnredlist.org, accessed in 18 ${ }^{\text {th }}$ July 2019.

JENKINS CN, ALVES MAS, UEZU A \& VALE MM. 2015. Patterns of vertebrate diversity and protection in Brazil. PLOS ONE 10: 1-13.

JOLY CA, METZGER JP \& TABARELLI M. 2014. Experiences from the Brazilian Atlantic Forest:ecological findings and conservation initiatives. New Phytologist 204: 459-473.

JORGE JS \& FREIRE EMX. 2011. Geographic Distribution: Thamnodynastes almae (Jararaca false snake). Herp Rev 42: 396.

JORGE JS, SALES RFD, SANTOS RL \& FREIRE ELX. 2020. Living among thorns: herpetofaunal community (Anura and Squamata) associated to the rupicolous bromeliad Encholirium spectabile (Pitcairnioideae) in Brazilian semi-arid Caatinga. Zoologia 37: e46661.

LIMA-VERDE JS. 1971. Fisioecologia e etologia de algumas serpentes da Chapada do Apodi, Estados do Ceará e Rio Grande do Norte (Brasil). Bol. Zool. Biol. Marinha 28: 189-238.

LIMA-VERDE JS. 1976. Fisioecologia e etologia de algumas serpentes da Chapada do Apodi, Estados do Ceará e Rio Grande do Norte (Brasil). Caatinga 1: 21-56.

LION MB, GARDA AA, SANTANA DJ \& FONSECA CR. 2016. The conservation value of small fragments for Atlantic Forest reptiles. Biotropica 48: 265-275.
LOEBMANN D \& HADDAD CFB. 2010. Amphibians and reptiles from a highly diverse area of the Caatinga domain: composition and conservation implications. Biota Neotrop 10: 227-256.

LOMOLINO MV. 2004. Conservation biogeography. In: Lomolino MV \& Heaney LR (Eds), Frontiers of Biogeography: New directions in the geography of nature, Sunderland: Sinauer Associates, Sunderland, England, p. 293-296.

MARQUES R, MEBERT K, FONSECA É, RÖDDER D, SOLÉ M \& TINÔCO MS. 2016. Composition and natural history notes of the coastal snake assemblage from Northern Bahia, Brazil. ZooKeys 2016: 93-142.

MARTINS M \& OLIVEIRA ME. 1998. Natural history of snakes in forests in the Manaus region, central Amazonia, Brazil. Herpetol Nat Hist 6: 78-150.

MEIRI S \& CHAPPLE DG. 2016. Biases in the current knowledge of threat status in lizards, and bridging the ‘assessment gap’. Biol Conserv 204: 6-15.

MERREM B. 1820. Versuch eines Systems der Amphibien I (Tentamen Systematis Amphibiorum). J. C. Kriegeri, Marburg.

MESQUITA ET AL. 2018. Herpetofauna in two habitat types (tabuleiros and Stational Semidecidual Forest) in the reserva Biológica Guaribas, northeastern Brazil. Herpetol Notes 11: 455-474.

MESQUITA PCMD, PASSOS DC, BORGES-NOJOSA DM \& CECHIN SZ. 2013. Ecologia e história natural das serpentes de uma área de Caatinga no nordeste brasileiro. Pap Avul Zool 53: 99-113.

MESQUITA PCMD, BORGES-NOJOSA DM, PASSOS DC \& BEZERRA CH. 2011. Ecology of Philodryas nattereri in the Brazilian semi-arid region. Herpetol J 21: 193-198.

MESQUITA PCMD, BORGES-NOJOSA DM, PASSOS DC \& BEZERRA CH. 2012. Activity patterns of the Brown Vine snake Oxybelis aeneus (Wagler, 1824) (Serpentes, Colubridae) in the Brazilian semiarid. Anim. Biol. 62: 289-299.

MEYER C, KREFT H, GURALNICK R \& JETZ W. 2015. Global priorities for an effective information basis of biodiversity distributions. Nat Commun 6: 8221.

MIRANDA JP, COSTA JCL \& ROCHA CFD. 2012. Reptiles from Lençóis Maranhenses National Park, Maranhão, northeastern Brazil. ZooKeys 246: 51-68.

MONTINGELLI GG, GRAZZIOTIN FG, BATTILANA J, MURPHY RW, ZHANG YP \& ZAHER H. 2019. Higher-level phylogenetic affinities of the Neotropical genus Mastigodryas Amaral, 1934 (Serpentes: Colubridae), species-group definition 
and description of a new genus for Mastigodryas bifossatus. J Zool Syst Evol Res 57: 1-35.

MOURA MR, VILLALOBOS F, COSTA GC \& GARCIA PCA. 2016. Disentangling the Role of Climate, Topography and Vegetation in Species Richness Gradients. PloS One 11: e0152468.

MOURA MR, PIRANI RM \& SILVA VX. 2013. New records of snakes (Reptilia: Squamata) in Minas Gerais, Brazil. Check List 9: 099-103.

MYERS CW \& CADLE JE. 1994. A New Genus for South American Snakes Related to Rhadinaea obtusa Cope (Colubridae) and Resurrection of Taeniophallus Cope for the "Rhadinaea" brevirostris Group. Am Mus Novit 3102: 1-33.

NASCIMENTO WS, BARROS NHC, ARAÚJO AS, GURGEL LL, CANAN B, MOLINA WF, ROSA RS \& CHELLAPPA S. 2014. Composição da Ictiofauna das Bacias Hidrográficas do Rio Grande do Norte, Brasil. Biota Amazônia 4: 126-131.

OLIVEIRA ET AL. 2016. The strong influence of collection bias on biodiversity knowledge shortfalls of Brazilian terrestrial biodiversity. Divers Distrib 22: 1232-1244.

OLSON ET AL. 2001. Terrestrial Ecoregions of the World: A New Map of Life on Earth. BioScience 51: 933.

PAREDERO RCB \& PASSOS MA. 2020. Chromatic anomaly in Apostolepis cearensis Gomes, 1915: First record of anerythrism in Elapomorphini. Herpetol Notes 13: 293-295.

PASSOS P \& FERNANDES R. 2008. Revision of the Epicrates cenchria Complex (Serpentes: Boidae). Herpetol Monogr 22: $1-30$.

PASSOS P, PRUDENTE ALC \& LYNCH JD. 2016. Redescription of Atractus punctiventris and description of two new Atractus (Serpentes: Dipsadidae) from Brazilian Amazonia. Herpetolol Monogr 30: 1-20.

PEEL MC, FINLAYSON BL \& MCMAHON TA. 2007. Updated world map of the Köppen-Geiger climate classification. Hydrol Earth Syst Sc 11: 1633-1644.

PEREIRA-FILHO GA \& MONTINGELLI GG. 2011. Check list of snakes from the Brejos de Altitude of Paraíba and Pernambuco, Brazil. Biota Neotrop 11: 145-151.

PEREIRA-FILHO GA, VIEIRA WLS, NÓBREGA RR \& FRANÇA F. 2017. Serpentes da Paraíba: Diversidade e conservação. João Pessoa: Gentil Alves Pereira Filho, 316 p.

PEREIRA ET AL. 2010. Scenarios for global biodiversity in the $21^{\text {st }}$ century. Science 330: 1496-1501.
PIRES MG, SILVA JR. NJ, FEITOSA DT, PRUDENTE ALC, PEREIRAFILHO GA \& ZAHER H. 2014. A new species of triadal coral snake of the genus Micrurus Wagler, 1824 (Serpentes: Elapidae) from northeastern Brazil. Zootaxa 3811: 569.

PIZZATTO L. 2005. Body size, reproductive biology and abundance of the rare Pseudoboini snakes genera Clelia and Boiruna (Serpentes, Colubridae) in Brazil. Phyllomedusa 4: 111-122.

RIBEIRO MM, LIMA GST, OLIVEIRA DV \& FREIRE EMX. 2014. Leptophis ahaetulla (Parrot Snake) Diet. Herpetol Rev 45: 339-340.

ROBERTO IJ \& LOEBMANN D. 2016. Composition, distribution patterns, and conservation priority areas for the herpetofauna of the state of Ceará, northeastern Brazil. Salamandra 52: 134-152.

ROSÁRIO IR, SANTOS RML, ARIAS F, ROCHA CFD, DIAS EJR, CARVALHO CM \& RODRIGUES MT. 2019. Phylogeography of the endangered sand dune whiptail lizard Glaucomastix abaetensis (Dias, Rocha \& Vrcibradic, 2002) with the description of a new species. Zootaxa 4624: 451-477.

RULL V. 2011. Neotropical biodiversity: Timing and potential drivers. Trends Ecol Evol 26: 508-513.

SALES RFD, LISBOA CMCA \& FREIRE E.M.X. 2009. Répteis Squamata de remanescentes florestais do Campus da Universidade Federal do Rio Grande do Norte, Natal- RN, Brasil. Cuad Herpetol 23: 77-88.

SALES RFD, JORGE JS, KOKUBUM MNC \& FREIRE EMX. 2013. Leptodeira annulata (Banded cat-eyed snake). Diet. Herpetol Rev 44: 524-525.

SAMPAIO ILR, SANTOS CP, FRANÇA RC, PEDROSA IMMC, SOLÉ M \& FRANÇA FGR. 2018. Ecological diversity of a snake assemblage from the Atlantic Forest at the south coast of Paraíba, northeast Brazil. ZooKeys 787: 107-125.

SANTANA GG, VIEIRA WLS, PEREIRA-FILHO GA, LIMA YCC \& VIEIRA KS. 2008. Herpetofauna em um fragmento de Floresta Atlântica no Estado da Paraíba, Região Nordeste do Brasil. Biotemas 21: 75-84.

SANTOS JR DL, SALES RFD, JORGE, JS \& FREIRE EMX. 2020. New record of Hydrodynastes gigas (Duméril, Bibron \& Duméril, 1854) (Serpentes, Dipsadinae) in northeastern Brazil. Check List 16: 457-460.

SCHIPPER ET AL. 2008. The Status of the World's Land and Marine Mammals: Diversity, Threat, and Knowledge. Science 322: 225-230.

SCHMIDT KP \& INGER RF. 1951. Amphibians and reptiles of Hopkins-Branner expedition to Brazil. Fieldiana Zool 31: 439-465. 
SFB - SERVIÇO FLORESTAL BRASILEIRO. 2018. Inventário Florestal Nacional: Principais Resultados: Rio Grande do Norte. Brasília: MMA, 65 p.

SOUZA PAG \& FREIRE EMX. 2008. Philodryas patagoniensis (NCN). Geographic distribution. Herpetol Rev 39: 243.

TAVARES AV, ARAÚJO KAM, MARQUES MRV, VIEIRA AA \& LEITE RS. 2017. The epidemiology of snakebite in the Rio Grande do Norte state, Northeastern Brazil. Rev I Med Trop 59: $1-10$.

TINGLEY R, MEIRI S \& CHAPPLE DG. 2016. Addressing knowledge gaps in reptile conservation. Biol Conserv 204: 1-5.

TONINI JFR, BEARD KH, FERREIRA RB, JETZ W \& PYRON RA. 2016. Fully-sampled phylogenies of squamates reveal evolutionary patterns in threat status. Biol Conserv 204: 23-31.

UETZ P, FREED P \& HOŠEK J. 2020. The Reptile Database, http://www.reptile-database.org, accessed $19^{\text {th }}$ May 2020.

VANZOLINI PE, RAMOS-COSTA AM \& VITT L. 1980. Répteis das Caatingas. Academia Brasileira de Ciências, Rio de Janeiro, Rio de Janeiro.

WALLACH V, WILLIAMS KL \& BOUNDY J. 2014. Snakes of the World: A Catalogue of Living and Extinct Species. Boca Raton: CRC Press, 1237 p.

\section{SUPPLEMENTARY MATERIAL}

Table SI. Detailed distribution of snakes in RN state with the recorded municipality of each species and the source material and voucher.

Figure S1. Geographic distribution maps of snake species and soil cover in Rio Grande do Norte State, Brazil. Leptotyphlopidae: (a) Epictia borapeliotes; Typhlopidae: (b) Amerotyphlops amoipira and A. paucisquamus; Boidae: (c) Boa constrictor, (d) Epicrates assisi and Corallus hortulana; Viperidae and Elapidae: (e) Bothrops erythromelas and B. leucurus, (f) Crotalus durissus, Micrurus potyguara and $M$. cf. corallinus. (g) M. aff. ibiboboca, and Colubridae: (h) Chironius flavolineatus and Palusophis bifossatus. Stars indicate new state records.

Figure S2. Geographic distribution maps of snake species and soil cover in Rio Grande do Norte State, Brazil. Colubridae and Dipsadidae: (a) Drymarchon corais, Apostolepis longicaudata and Erythrolamprus mossoroensis, (b) Leptophis ahaetulla, (c) Oxybelis aeneus, (d) Tantilla melanocephala, (f) Apostolepis cearensis and Erythrolamprus almadensis, (g) Dipsas mikanii and Helicops angulatus, and (h) Imantodes cenchoa, Hydrodynastes gigas and Erythrolamprus miliaris. Stars indicate new state records.

Figure S3. Geographic distribution maps of snake species and soil cover in Rio Grande do Norte State, Brazil. Colubridae and Dipsadidae: (a) Erythrolamprus poecilogyrus and Psomophis joberti, (b) E. viridis, (c) Helicops leopardinus, Leptodeira annulata, and Sibon nebulatus, (d) Lygophis dilepis, (e) Oxyrhopus trigeminus, (f) Philodryas nattereri, (G) P. olfersii, and (H) Phimophis guerini and Philodryas patagoniensis. Figure S4. Geographic distribution maps of snake species and soil cover in Rio Grande do Norte State, Brazil. Dipsadidae: (a) Pseudoboa nigra, (b) Taeniophallus occipitalis, (c) Thamnodynastes almae, T. phoenix and T. sertanejo, and (d) Xenodon merremii. Stars indicate new state records.

How to cite

MARQUES R, GUEDES TB, LANNA FM, PASSOS DC, SILVA WP \& GARDA AA. 2021. Species richness and distribution patterns of the snake fauna of Rio Grande do Norte state, northeastern Brazil. An Acad Bras Cienc 93: e20191265. DOI 10.1590/0001-3765202120191265.

Manuscript received on October 19, 2019;

accepted for publication on July 5, 2020

\section{RICARDO MARQUES ${ }^{1}$}

https://orcid.org/0000-0002-4632-8808

THAÍS BARRETO GUEDES ${ }^{2,3}$

https://orcid.org/0000-0003-3318-7193

FLÁVIA MOL LANNA ${ }^{4}$

https://orcid.org/0000-0002-7263-872X

DANIEL CUNHA PASSOS 5

https://orcid.org/0000-0002-4378-4496

WILLIANILSON PESSOA DA SILVA ${ }^{1}$

https://orcid.org/0000-0002-4975-5733

ADRIAN ANTONIO GARDA ${ }^{6}$

https://orcid.org/0000-0002-1178-1207 
'Universidade Federal da Paraíba, Programa de PósGraduação em Ciências Biológicas (Zoologia), Centro de Ciências Exatas e da Natureza, Departamento de Sistemática e Ecologia, Campus I - Cidade Universitaria, s/n, Cidade Universitária, 58051-900 João Pessoa, PB, Brazil ${ }^{2}$ Universidade Estadual do Maranhão, Programa de PósGraduação em Biodiversidade, Ambiente e Saúde, Centro de Estudos Superiores de Caxias, Praça Duque de Caxias, s/n, Morro do Alecrim, 65604-380 Caxias, MA, Brazil

${ }^{3}$ Gothenburg Global Biodiversity Center, Box 461, SE-405 30, Göteborg, Sweden

${ }^{4}$ The Ohio state University, Department of Evolution, Ecology, and Organismal Biology, 318 W. $12^{\text {th }}$ Ave, 43210, Columbus, OH, USA

${ }^{5}$ Universidade Federal Rural do Semi-Árido, Laboratório de Ecologia e Comportamento Animal, Programa de Pós-Graduação em Ecologia e Conservação, Departamento de Biociências, Centro de Ciências Biológicas e da Saúde, Avenida Francisco Mota, 572, Pres. Costa e Silva, 59625-900 Mossoró, RN, Brazil ${ }^{6}$ Universidade Federal do Rio Grande do Norte, Centro de Biociências, Departamento de Botânica e Zoologia, Avenida Senador Salgado Filho, s/n, Lagoa Nova, 59078-900 Natal, RN, Brazil

Correspondence to: Ricardo Marques

E-mail:rcdmarquess@gmail.com

\section{Author contributions}

RM designed the manuscript, reviewed the specimens housed at LAR-UFRN, gathered all data and performed data interpretation, elaborated figures of heat and distribution maps, tables, and wrote the manuscript. TBG helped in the conceptualization, gathered data, review the analyses, writing - original draft, review and edit final manuscript. FML gathered data from literature, elaborated figures and writing. DCP provided primary data, contributed to the writing of early versions and reviewed the final version of manuscript. WPS collected part of the data. AAG conceived the idea of the paper, collected field data, and participated in the production of the manuscript. All authors discussed and reviewed the final version of the manuscript.

\section{(c) BY}

